\title{
一种快速高效分离和纯化重组腺病毒伴随病毒 载体的方法
}

吴小兵 ${ }^{(1)}$ 董小岩 ${ }^{(1)}$ 伍志坚 ${ }^{(1)}$ 屈建国 ${ }^{(2)}$ 侯云德 $1{ }^{1}$ (中国预防医学科学院病毒学研究所(1)病毒基因工程国家重点实验室; (2)形态室, 北京 100052 .

*Email: wuxb@public.east.cn.net)

摘要 利用 2 型腺病毒伴随病毒(adeno-associated virus type 2, AAV-2)能够抵抗氯仿的特性, 采用 “氯仿处理-PEG/ $\mathrm{NaCl}$ 沉淀-氯仿抽提” 3 个步骤分离、浓缩和纯化重组腺病毒伴随病毒(recombinant adeno-associated virus, rAAV), 整个纯化过程可在 $4 \mathrm{~h}$ 内完成. 最终从 5 个转瓶生长的 $4 \times 10^{9}$ 个载 体生产细胞中可得到 $5 \times 10^{13}$ 病毒颗粒 $/ \mathrm{mL}$, SDS-聚丙烯酰胺凝胶结果表明, rAAV 的纯度大于 95\%, 电子显微镜下绝大多数病毒颗粒的结构完整. 纯化后的 rAAV 仍保持较强的感染性, 其感 染性滴度达到 $2 \times 10^{12}$ 转导单位 (transducing unit, TU)/mL, 总的病毒颗粒数与感染性病毒颗粒数 的比值为 25 . 为 $\mathrm{rAAV}$ 大规模生产后的纯化提供了一种简便、快速、低成本的方法.

关键词 重组腺病毒伴随病毒 氯仿 纯化

由 2 型腺病毒伴随病毒(AAV-2)改造而来的重组腺病毒伴随病毒(rAAV)是一种安全、有 效的基因转移载体，已用于多种人类疾病的基因治疗研究. 然而长期以来，大量、高纯度的 rAAV 一直难以获得, 使其应用受到了限制. 近两年来, rAAV 的大规模制备策略有了突破性进 展. 伍志坚和 Conway 等人 ${ }^{[1,2]}$ 先后构建了具有 rAAV 包装功能的重组 1 型单纯疮疹病毒 (recombinant herpes simplex virus type 1, rHSV-1), 以此感染携带 rAAV 前病毒的载体细胞株, 可方 便地实现 rAAV 的大规模生产. 但是, 在 rAAV 的纯化方面目前仍然缺乏高效、简便、低成本的 方法. 常规的方法是采用硫酸铵浓缩、氯化铯密度梯度离心进行纯化 ${ }^{[3]}$, 这种方法费时、步骤繁 琐、回收率低. 由于已初步鉴定出硫酸乙酰肝素蛋白多糖(heparin sulfate proteoglycan)是细胞膜上 AAV-2 的受体 ${ }^{[4]}$, 特异性识别成熟 AAV-2 毒粒的单克隆抗体也已成功制备 ${ }^{[5]}$, 因此, 利用肝素亲 和层析 ${ }^{[6,7]}$ 或免疫亲和层析 ${ }^{[8]}$ 可以高效地纯化 $\mathrm{rAAV}$, 但这类方法费用较高, 不易推广. 本研究充分 利用了 AAV-2 的外壳能够抵抗氯仿的性质, 采用氯仿处理-PEG/ $\mathrm{NaCl}$ 沉淀-氯仿抽提 3 个步骤, 提供了一种快速、简便、高效地分离、浓缩和纯化 rAAV 的新方法.

\section{1 材料与方法}

（i ）主要实验材料. 含有绿色菼光蛋白 (green fluorescent protein, GFP)基因的 rAAV 载 体质粒 pSNAV-GFP 为我们课题组将 pGREEN-LANTERN(GIBCO/BRL)上的 GFP 基因插入 pSNAV 载体 ${ }^{[9]}$ 的多克隆位点构建而成(待发表资料). HeLa 细胞购自 ATCC. BHK/SG1 细胞为 我们课题组先前建立的含 GFP 基因表达盒的 AAV 载体细胞株. 上述细胞均用含 $10 \%$ 胎牛血清 的 RPMI 1640 培养液培养. 具有 AAV 复制和包装功能的重组 1 型单纯疮疹病毒一 HSV1$\mathrm{rc} / \Delta \mathrm{UL} 2$ 为我们先前重组产生 ${ }^{[1]}$, 在 BHK-21 细胞上传代、扩增. 用于细胞大规模培养的转瓶 $(110 \mathrm{~mm} \times 480 \mathrm{~mm})$ 及转瓶机为 Wheaton 公司产品.

(ii) 表达 GFP 的 rAAV(rAAV-GFP)的生产. 将 BHK/SG1 细胞扩大培养后接种到 5 个转 瓶中, 培养液体积为每个转瓶 $200 \mathrm{~mL}$. 细胞长满后(共约 $4 \times 10^{9}$ 个细胞)将培养液倾出, 加 $10 \mathrm{~mL}$ 
辅助病毒 HSV-rc/ $\Delta$ UL2 $(M O I=0.1)$, 低速转动吸附病毒 $2 \mathrm{~h}$. 每个转瓶加 $200 \mathrm{~mL}$ 无血清 1640 培养液 $37^{\circ} \mathrm{C}$ 低速转动培养. 为避免纯化时体积过大, $24 \sim 36 \mathrm{~h}$ 后, 将每个转瓶中的液体弃去 $100 \mathrm{~mL}$. 继续培养 24 48 h 直到细胞完全病变、容易脱落时, 盖紧瓶盖剧烈振摇, 将瓶壁上的细胞全 部洗脱至培养液中. 收集合并 5 个转瓶的培养物.

(iii) rAAV 的纯化. 步骤见图 1. 将收集的培养物定义为初始物. 加入 $1 / 10$ 体积的氯仿, 置于 $37^{\circ} \mathrm{C}$ 摇床中剧烈振摇 $1 \mathrm{~h}$, 加入固体氯化钠至终浓度 $1 \mathrm{~mol} / \mathrm{L}$, 振摇溶解. $4{ }^{\circ} \mathrm{C}, 12000 \mathrm{r} / \mathrm{min}$ 离心 $15 \mathrm{~min}$. 取出上层水相, 弃去氯仿和沉淀. 加 PEG8000 至终浓度 $10 \%(\mathrm{w} / \mathrm{v})$, 振摇溶解后 冰浴放置 $1 \mathrm{~h}, 11000 \mathrm{r} / \mathrm{min}$ 离心 $15 \mathrm{~min}$. 将上清弃去, 用 $5 \mathrm{~mL} \mathrm{PBS}^{2+}$ 缓冲液将各离心管管底和 管壁上的沉淀吹打洗脱下来合并, 将其分装至 $1.5 \mathrm{~mL}$ 塑料离心管中 ( $0.6 \mathrm{~mL} /$ 管), 此时的 $\mathrm{rAAV}$ 定义为中间产物. 加入 DNase 和 RNase 至终浓度 $1 \mu \mathrm{g} / \mathrm{mL}$, 室温下消化 $30 \mathrm{~min}$. 加等体积的 氯仿抽提. $4^{\circ} \mathrm{C}, 12000 \mathrm{r} / \mathrm{min}$ 离心 $5 \mathrm{~min}$, 在无菌操作下小心吸出上层水相. 该液体即为浓缩和 纯化的 rAAV-GFP 病毒液, 定义为终产物. 总回收率 = 终产物的感染性病毒颗粒数/初始物 的感染性病毒颗粒数.

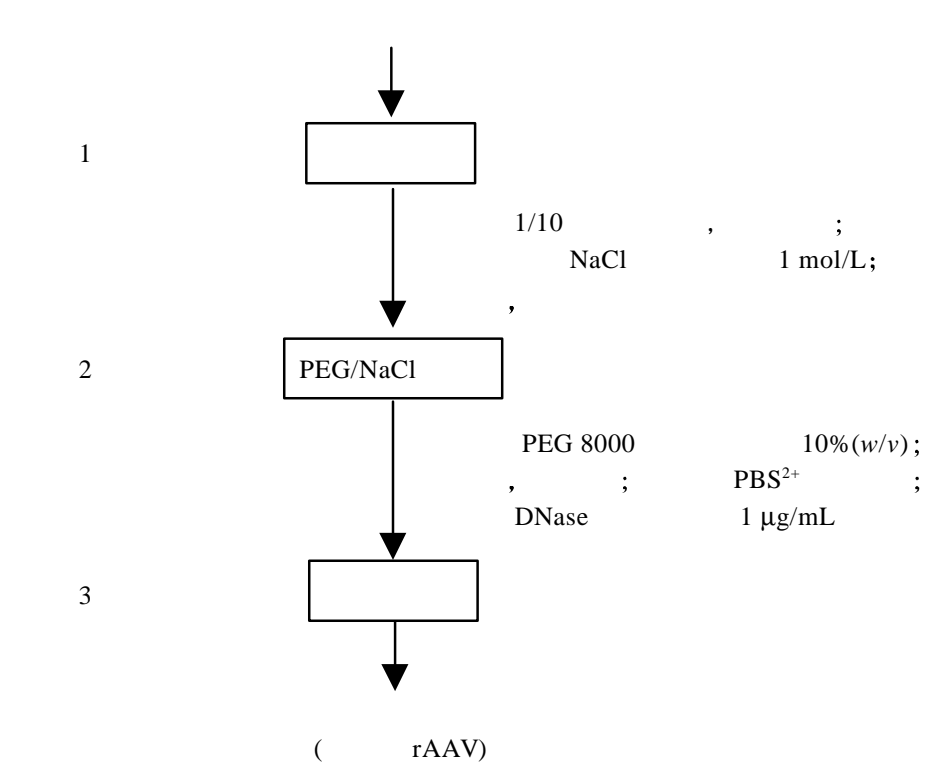

图 1 用三步法分离、浓缩、纯化 $\mathrm{rAAV}$ 的示意图

(iv) 用 SDS-聚丙烯酰胺凝胶(SDS-PAGE)检测 rAAV 的纯度. 按文献[9]方法灌制 SDSPAGE 分离胶和积层胶, 分离胶浓度为 $10 \%$. 分别取纯化的病毒液和最后一步氯仿抽提前的病 毒液 $20 \mu \mathrm{L}$, 加 $2 \times$ 加样缓冲液 $20 \mu \mathrm{L}$, 沸水浴 $3 \mathrm{~min}$. 每个加样孔加样 $10 \mu \mathrm{L}$. 电泳完毕后用 考马斯亮蓝染色, 用相应的脱色液脱色直到显出低背景、清晰的条带.

( V ) rAAV 病毒的电子显微镜分析. 纯化的 rAAV-GFP 经铇酸盐负染后, 置于 150 目的 镍网上, 电子显微镜观察.

(vi) rAAV 病毒物理滴度检测. 用地高辛标记(Boehinger Mannhein 试剂盒)的 GFP 探针 点杂交方法检测纯化的病毒液中 rAAV-GFP 的物理滴度 (病毒颗粒数/mL). 将质粒 pSNAV-GFP 准确定量, 用稀释缓冲液以一系列稀释度稀释后点到尼龙膜上. 取 $10 \mu \mathrm{L}$ 纯化的病毒液用 
$\mathrm{PBS}^{2+}$ 缓冲液稀释 10 倍, 加 DNase 和 RNase 至终浓度 $1 \mu \mathrm{g} / \mathrm{mL}, 37^{\circ} \mathrm{C}$ 消化 $1 \mathrm{~h}$. 沸水浴 $5 \mathrm{~min}$ 之后置于冰浴中. 用稀释缓冲液以 $1: 10$ 作系列稀释后点膜. $120^{\circ} \mathrm{C}$ 烤膜 $30 \mathrm{~min} .68^{\circ} \mathrm{C}$ 预杂交 $1 \mathrm{~h}$. 加探针 $68^{\circ} \mathrm{C}$ 杂交过夜, 洗膜, 显色. 通过计算 pSNAV-GFP 的分子数, 再乘以与其杂交信号强 度一致的病毒液样品的稀释倍数得出 rAAV-GFP 的物理滴度.

(vii) rAAV-GFP 感染性滴度的测定. 在 24 孔培养板上接种 HeLa 细胞, $5 \times 10^{5}$ 细胞/孔. 培 养过夜后, 吸出培养液; 取 $10 \mu \mathrm{L}$ 纯化的病毒液稀释至 $1 \mathrm{~mL}$, 以 $1: 10$ 作系列稀释, 每孔加 不同稀释度的病毒液 $0.5 \mathrm{~mL}, 37^{\circ} \mathrm{C}$ 培养 $1 \mathrm{~h}$. 每孔加 5 型腺病毒( $\left.\mathrm{Ad} 5\right) 50 \mu \mathrm{L}(\mathrm{MOI}=5)$ 及培养液 $0.5 \mathrm{~mL} .37^{\circ} \mathrm{C}$ 培养 $36 \mathrm{~h}$ 后在倒置苂光显微镜下观察绿色苂光细胞, 计数其中某孔的绿色细胞 数 $n(10<n<100)$. rAAV-GFP 病毒滴度(转导单位 $/ \mathrm{mL})=n \times$ 稀释倍数 $\times 1000 / 5$.

\section{2 结果}

\section{1 rAAV-GFP 的分离、浓缩与纯化}

纯化步骤的流程图见图 1, 主要包括氯仿处理-PEG/NaCl 沉淀-氯仿抽提 3 个步骤. 纯化的终 产物用 $5 \mathrm{~mL} \mathrm{PBS}^{2+}$ 即可溶解, 与 $500 \mathrm{~mL}$ 的起始物相比浓缩至原体积的 $1 / 100$, 总回收率约为 $90 \%$.

\section{2 rAAV-GFP 的纯度}

AAV-2 的外壳由 3 种蛋白质组成, 即 VP1, $\mathrm{VP} 2, \mathrm{VP} 3$, 分子量依次为 $87,72,62 \mathrm{ku} . \mathrm{AAV}$ 病毒 颗粒中 VP1, VP2, VP3 蛋白的比例约为 $10: 1: 1$, 在 SDS-PAGE 电泳后形成 3 条特征性条带. 用 SDS-PAGE 分析了不同阶段 rAAV- GFP 的纯度, 结果见图 2. 在 $\mathrm{rAAV}$ 的终产物(泳道 2)和中间产 物(泳道 3)中均可见到代表 AAV 外壳蛋白 VP1, VP2，VP3 的 3 条带，在中间产物中还可见到各种 分子量的其他蛋白质, 而终产物的背景则非常低, 这表明氯仿抽提可以有效地去除杂蛋白, 而 rAAV 没有明显的损失. 经计算机扫描分析，在 rAAVGFP 的终产物中, rAAV-GFP 的纯度大于 $95 \%$. 在 等体积未经浓缩的 rAAV-GFP 起始物中未见明显 的外壳蛋白. 需要指出的是, 与其他研究者所采

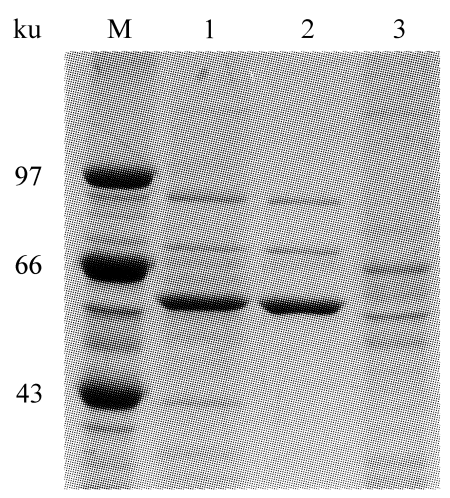

图 2 rAAV-GFP 纯化的不同阶段 SDS-PAGE 考马斯亮蓝的染色结果

$M$ 为分子量标准蛋白 (Promega); 1 为末经纯化的 rAAV-GFP(从 $500 \mathrm{~mL}$ 起始物中取 $5 \mu \mathrm{L}$ ); 2 为纯化的 rAAV-GFP(从 $5 \mathrm{~mL}$ 终产物中取 $5 \mu \mathrm{L}) ; 3$ 为未经最后 一步抽提的 rAAV-GFP(从 $5 \mathrm{~mL}$ 中间产物中取 $5 \mu \mathrm{L}$ ) 用的银染或 Western 印迹分析方法相比，本文采用的考马斯亮蓝染色方法的灵敏度较低，提示 检测到的外壳蛋白浓度相当高.

\section{3 纯化后 rAAV-GFP 的电子显微镜分析}

rAAV-GFP 经负染后用电子显微镜观察, 结果见图 3. 大量的 rAAV 颗粒清晰可见, 绝大 多数病毒颗粒是完整的, 少数颗粒是病毒装配的中间形式, 未见病毒的空壳形式. 结果表明, 纯化后的 rAAV-GFP 终产物中含有高浓度、高纯度的 rAAV 颗粒.

\section{4 纯化后 rAAV-GFP 的滴度}

用地高辛标记的 GFP 基因作探针, 点杂交检测了病毒颗粒中的 rAAV 基因组. 纯化后 
rAAV-GFP 的物理滴度约为 $5 \times 10^{13}$ 病毒颗粒 $/ \mathrm{mL}$. 用转基因表达的方法测定纯化后 rAAVGFP 的感染性滴度约为 $2 \times 10^{12}$ 转导单位 $/ \mathrm{mL}$. 病毒颗粒总数与感染性病毒的比例为 25 , 说 明经过一系列处理后, rAAV 的感染能力未见 明显丧失.

\section{3 讨论}

由于 $\mathrm{rAAV}$ 在基因治疗研究中的地位正在 日益提高, 有关 rAAV 规模化生产的策略研究 屡见报道, 然而对于 rAAV 纯化方法的研究却 不多见. 随着 rAAV 大规模生产技术的成熟, 解决 rAAV 的纯化问题显得尤为重要. 传统的

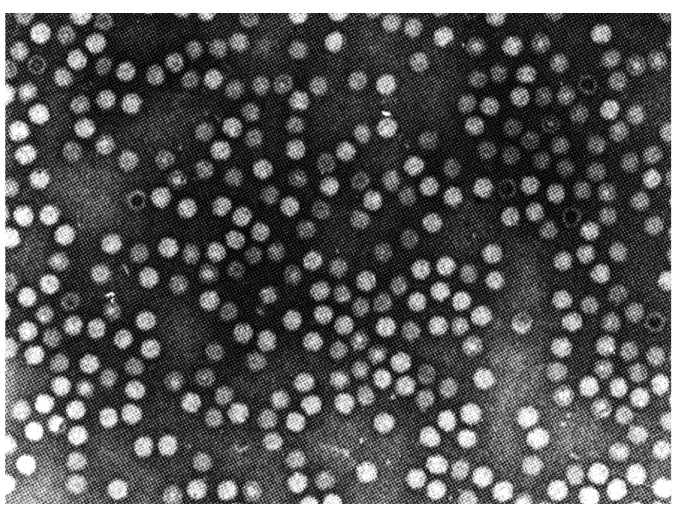

图 3 纯化的 rAAV-GFP 的电子显微镜分析 $(\times 54800)$ 纯化方法 ${ }^{[3]}$ 采用硫酸铵分步盐析法将 $\mathrm{rAAV}$ 与细胞碎片分离并使之浓缩, 然后用 $2 \sim 3$ 轮氯化 铯梯度超离心获得 rAAV 组分, 最后用透析方法除去氯化铯和其他盐类. 这种方法费时费力、 回收率低, 同时 rAAV 的感染性也有较大的损失, 而且, 氯化铯对人体具有潜在的毒性, 因此, 这种方法已逐渐被淘汰. 许多研究者致力于发展新的纯化方法. Grimm 等人 ${ }^{[8]}$ 利用能特异性识 别成熟 AAV 毒粒的单克隆抗体 $\mathrm{A} 20^{[5]}$, 发展了一种免疫亲和柱层析的 rAAV 纯化方法, 能在 一个工作日内完成纯化步骤, 回收率达到 70\%, 纯度达到 80\%. 由于肝素是 AAV 天然受体的 类似物 ${ }^{[4]}$, 另外两个实验室分别发展了肝素亲和层析的纯化方法. 采用这种纯化方法的潜在问 题是很多细胞蛋白质也同肝素结合, 因此需要解决如何去除杂蛋白的问题. Zolotukhin 等人 ${ }^{[7]}$ 采取的办法是先用碘狄醇密度梯度离心, 得到“半纯化”的 rAAV, 再用肝素亲和层析进行纯化, 可使回收率达到 50\% 70\%, 纯度可达 $99 \%$. Clark 等人 ${ }^{[6]}$ 则先用脱氧胆酸裂解细胞, 离心得到 上清后 $56^{\circ} \mathrm{C}$ 加热 $45 \mathrm{~min}$ 灭活辅助病毒. 脱氧胆酸裂解与热处理可使蛋白变性形成絮状沉淀, 离心去除沉淀后再进行肝素亲和层析, 结果表明这种方法回收率高于 $70 \%$, 纯度可达 $99 \%$. 尽 管这些方法使 rAAV 的纯化过程大大简化, 回收率有较大提高, 但亦有成本高、处理大体积 样品能力有限、实验条件和仪器设备要求高等缺点, 因此仍需寻找快速、简便、高效、低成 本的纯化方法.

AAV-2 的外壳对氯仿具有天然的抵抗性, 而 $\mathrm{rAAV}$ 与 AAV-2 的外壳完全一样, 因此氯仿 有可能成为分离和纯化 $\mathrm{rAAV}$ 的有效工具. 我们参照了 $\lambda$ 噬菌体的分离和纯化方法 ${ }^{[10]}$ 并加以 改进, 提出了“氯仿处理-PEG/NaCl 沉淀-氯仿抽提”的 $\mathrm{rAAV}$ 纯化方法. 第 1 步氯仿处理可达 到 3 个目的: ( i ) 可彻底灭活辅助病毒, 同时免去了热灭活 $\left(56^{\circ} \mathrm{C}, 30 \sim 60 \mathrm{~min}\right)$ 的步骤; (ii) 可 以很容易地裂解细胞, 与常规的方法 (反复冻融 3 次)相比, rAAV 的释放更快速、有效; (iii) 可 使大量的细胞蛋白质变性、沉淀, 易于离心除去. 第 2 步采用 $1 \mathrm{~mol} / \mathrm{L} \mathrm{NaCl} / 10 \% \mathrm{PEG}$ 代替硫 酸铵对 $\mathrm{rAAV}$ 进行浓缩, 可使 $\mathrm{rAAV}$ 浓缩至原体积的 $1 / 100$. 最后一步氯仿抽提可非常有效地 除去残留的 PEG 及不需要的蛋白质, 同时并不损失 rAAV. 整个纯化过程在 $4 \mathrm{~h}$ 内即可完成, 纯化后的 $\mathrm{rAAV}$ 终产物可在 $4^{\circ} \mathrm{C}$ 保存 1 个月以上而滴度没有明显降低. 残留的少量氯仿可通 过生物化学方法进一步除去. 
与传统的氯化铯密度梯度离心方法相比, 本方法快速、回收率高, 同时不降低 rAAV 的 感染能力. 与最近发展起来的几种柱层析方法相比, 本方法成本低廉, 在工艺上有明显优势. 用本方法获得的 rAAV 可用于基因治疗的体外实验和动物实验, 进一步纯化后可获得临床级 的 AAV 产品. 该方法适合于大规模分离和纯化 rAAV 病毒, 并使其生产工艺化, 尤其适用于 以 HSV 病毒为辅助病毒产生的 rAAV 的纯化, 也可用于无辅助病毒包装系统或无细胞体外包 装系统生产的 rAAV 的纯化.

\section{参 考 文 献}

1 伍志坚, 吴小兵, 侯云德. 具有 AAV 载体包装功能的重组 HSV 的产生. 科学通报, 1999, 44(5): 506 509

2 Conway J E, Rhys C M J ap, Zolotukhin I, et al. High-titer recombinant adeno-associated virus production utilizing a recombinant herpes simplex virus type 1 vector expressing AAV-2 Rep and Cap. Gene Therapy, 1999, 6: 986 993

3 Synder R O, Xiao X, Samulski R J. Production of recombinant adeno-associated viral vectors. In: Dracopoli N, ed. Current Protocols in Human Genetics. New York: John Wiley, 1996

4 Summerford C, Samuski R J. Membrane-associated heparan sulfate proteoglycan is a receptor for high-titer recombinant adeno-associated virus type 2 virions. J Virol, 1998, 72: 1438 1445

5 Wistuba A, Kern A, Weger S, et al. Subcellular compartmentalization of adeno-associated virus type 2 virions. J Virol, 1997, 71: $1341 \sim 1352$

6 Clark K R, Liu X, McGrath J P, et al. Highly purified recombinant adeno-associated virus vectors are biologically active and free of detectable helper and wild-type viruses. Hum Gene Ther, 1999, 10: 1031 1039

7 Zolotukhin S, Byrne B, Mason E, et al. Recombinant adeno-associated virus purification using novel methods improves infectious titer and yield. Gene Therapy, 1999, 6: 973 985

8 Grimm D, Kern A, Rittner K, et al. Novel tools for production and purification of recombinant adenoassociated virus vectors. Hum Gene Ther, 1998, 9: 2745 2760

9 伍志坚, 吴小兵, 侯云德. 系列腺病毒伴随病毒载体的构建及表达 $\beta$-半乳糖苷酶的研究. 病毒学报, 2000, 16(1): 1 6

10 Sambrook J, Fritsch E F, Maniatis, et al. Molecular Cloning: A Laboratory Manual. 2nd ed, New York: Cold Spring Harbour Press, 1989

(2000-03-09 收稿, 2000-06-19 收修改稿) 